06

\title{
Рентгеновская диагностика дефектов микроструктуры кристаллов кремния, облученных ионами водорода
}

\author{
() В.Е. Асадчиков, И.Г. Дьячкова, Д.А. Золотов, Ю.С. Кривоносов, Ф.Н. Чуховский \\ Институт кристаллограсрии им. А.В. Шубникова РАН, \\ Федеральный научно-исследовательский центр „Кристаллография и фотоника“ РАН, \\ 119333 Москва, Россия \\ e-mail: sig74@mail.ru
}

Поступило в Редакцию 25 сентября 2018 г.

В окончательной редакции 25 сентября 2018 г.

Принято к публикации 23 октября 2018 г.

\begin{abstract}
Изучены особенности образования и трансформации радиационных дефектов в приповерхностных слоях кремниевых пластин, имплантированных ионами водорода. Используя метод рентгеновской двухкристальной дифрактометрии высокого разрешения, определены значения основных параметров, таких как средняя эффективная толщина $L_{\text {eff }}$ и средняя относительная деформация $\Delta a / a$ легированного слоя в зависимости от дозы имплантации и температуры подложки.
\end{abstract}

DOI: 10.21883/JTF.2019.05.47476.346-18

\section{Введение}

Современная технология производства полупроводниковых приборов микроэлектроники, в основе создания которых лежит ионное легирование поверхностного слоя подложки, достигла уровня исполнения, при котором существенным фактором является образование дефектов, привнесенных туда пучком протонов и, как результат, их влияние на физико-химические характеристики кремниевых подложек [1].

Микродефекты (МД), которые образуются в результате коагуляции точечных дефектов, создают относительно сильные поля упругих искажений и приводят к латеральной неоднородности и изменениям свойств подложки [2]. Исследование свойств дефектов, возникающих при ионной имплантации, позволит расширить возможности современной технологии создания новых приборов и управления характеристиками микроэлектронных устройств.

Диагностика структурного совершенства ионноимплантированных образцов кремния является важным фактором подбора технологического режима имплантации с целью получения контролируемых свойств нарушенного слоя с точки зрения решения определенных практических задач.

В настоящей работе с использованием метода рентгеновской двухкристальной дифрактометрии высокого разрешения определены интегральные характеристики кристаллических слоев кремния под действием имплантации протонов, выявлена зависимость характера дефектообразования в них в зависимости от дозы ионной имплантации и температуры подложки.

\section{Приготовление образцов и методика эксперимента}

Для выявления влияния дозы и температуры протонного облучения на характеристики нарушенного слоя были приготовлены образцы кристаллов кремния КЭФ-4.5, с ориентацией поверхности (100), толщина $400 \mu \mathrm{m}$. Эти образцы облучались ионами водорода с энергией $150 \mathrm{keV}$ при различных значениях дозы облучения $2.5 \cdot 10^{15}, 5 \cdot 10^{15}, 1 \cdot 10^{16}, 2 \cdot 10^{16} \mathrm{~cm}^{-2}$. Облучение образцов проводилось на ускорители КГ-500 НИИЯФ МГУ [3] при различных температурах 50, 140, 230, 320, $430,550,610^{\circ} \mathrm{C}$.

Исследования имплантированных образцов кремния проводились на двухкристальном рентгеновском спектрометре в бездисперсионной схеме с использованием отражения четвертого порядка от кристаллографических плоскостей $(100)$, угол Брэгга $\theta_{\mathrm{B}}=34.5^{\circ}$. Использовалось $\mathrm{Cu} k_{\alpha 1}$-излучение, $\lambda=1.541 \AA$, при ускоряющем напряжении $25 \mathrm{keV}$ и токе $5 \mathrm{~mA}$ рентгеновской трубки. Во всех экспериментах характеристическая линия падающего на кристалл-образец излучения $\mathrm{Cu} k_{\alpha 2}$ обрезалась коллимационной щелевой системой, не оказывая, в пределах статистической погрешности, влияния на измеряемые КДО во всем диапазоне углов вращения образца. Глубина экстинкции $L_{\mathrm{ext}}$, характеризующая дифракционную глубину проникновения рентгеновских лучей в кристалл, в нашем эксперименте составила $3.6038 \mu \mathrm{m}$. Для пространственной коллимации падающего на образец рентгеновского излучения использовались ограничивающие „крест-на-крест“ щели в горизонтальной и вертикальной плоскостях, ширины которых составляли 0.1 и $12 \mathrm{~mm}$ соответственно.

Для всех приготовленных образцов были получены кривые дифракционного отражения (КДО). Измерение КДО осуществлялось в режиме автоматического вращения исследуемых образцов кристаллического кремния в определенном интервале вблизи угла Брэгга с шагом $0.5 \operatorname{arcsec}$ и временем счета $1 \mathrm{~s}$ на одну точку КДО.

Из экспериментальных КДО рассчитывались величины эффективной толщины $L_{\mathrm{eff}}$ и средней относительной деформации кристаллической решетки дефектного слоя $\Delta a / a$ по методу интегральных характеристик [4]. 


\section{Результаты и их обсуждение}

На рис. 1 представлена КДО исходного образца, не подвергавшегося никаким видам воздействия. Полуширина кривой качания составляет около $3 \mathrm{arcsec}$, что свидетельствует о том, что исходный кристалл кремния является высокосовершенным и содержит минимальное количество дефектов. Характер снятых КДО для всех доз имплантации водорода в кремний изменился по отношению к КДО от необлученного кристалла, а именно появились (помимо основного максимума) дополнительные осцилляции интенсивности, характеризующие образование нарушенного слоя (рис. 2,3). На рис. 2 и 3 основной пик КДО от ненарушенного ионной имплантацией слоя кремниевой пластины обрезан для увеличения изображения области дополнительной интенсивности. Во всех случаях КДО ассиметричны, причем со стороны углов, меньших угла Брэгга, интенсивность больше, чем интенсивность с противоположной стороны. Кроме того, наблюдается появление дополнительного максимума, обусловленного структурой нарушенного в результате имплантации слоя. Для всех указанных доз имплантации при температурах от 50 до $550^{\circ} \mathrm{C}$, при явно выраженном пике от нарушенного слоя, хорошо заметны когерентные осцилляции со стороны малых углов, характерные для деформации $\Delta a / a$ со знаком плюс, обусловленной образованием дефектов междоузельного типа [4,5]. Из сравнения рис. $2, a, b$ и $3, a, b$ следует, что с увеличением дозы имплантации (ДИ) имеет место „дополнительное“ рассеяние, приводящее к увеличению значений интенсивности на КДО.

Видно, что с увеличением температуры от 50 до $430^{\circ} \mathrm{C}$ период осцилляций на КДО уменьшается, а область дополнительной интенсивности увеличивается (рис. $2, a, b$ и $3, a, b$ ), что свидетельствует об увеличении толщины $L_{\mathrm{eff}}$ и деформации $\Delta a / a$ нарушенного слоя.

При температуре $430^{\circ} \mathrm{C}$ отмечен резкий рост КДО от нарушенного слоя (рис. 2, $b$, и $3, b$ ). С дальнейшим увеличением температуры границы этой области сужаются, а сам пик нарушенного слоя резко приближается

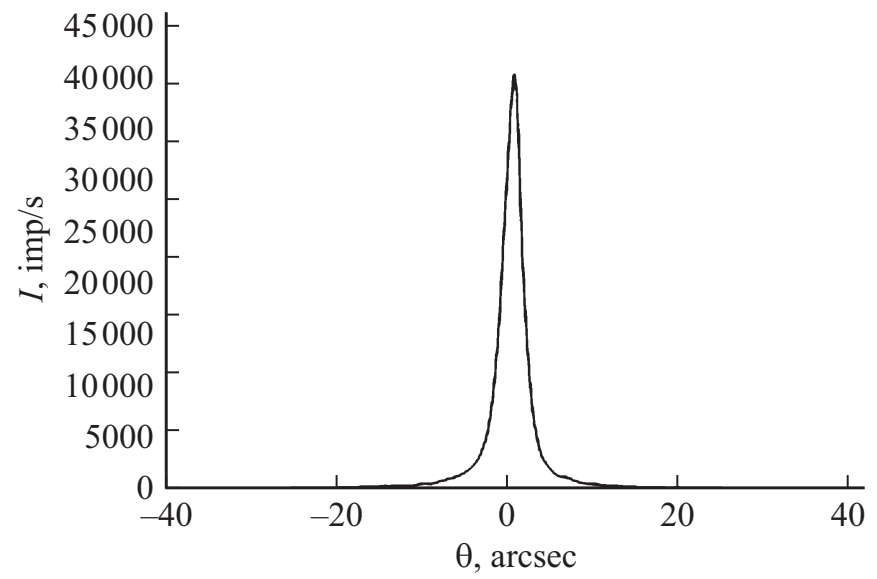

Рис. 1. КДО от необлученного образца $\mathrm{Si}$.
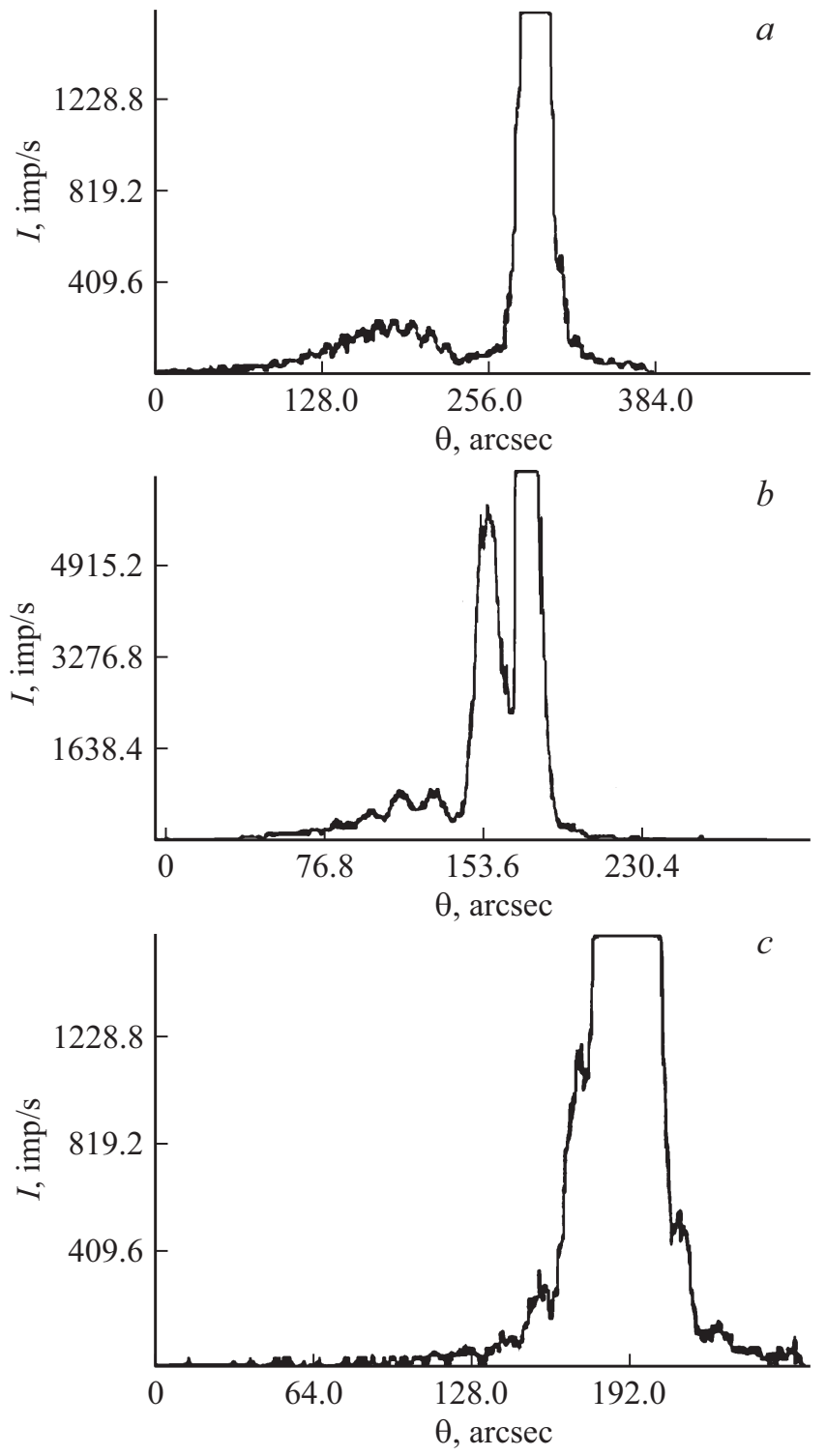

Рис. 2. КДО от образцов $\mathrm{Si}$, облученных протонами с дозой $5 \cdot 10^{15} \mathrm{~cm}^{-2}$ при различных значениях температуры: $a-140$, $b-430, c-610^{\circ} \mathrm{C}$.

к пику подложки (рис. 2, c,и 3, c), что свидетельствует об уменьшении деформации $(\Delta a / a)$ в нарушенном слое.

На представленных рисунках видно, что на КДО со стороны углов, больших угла Брэгга, также обнаруживаются слабые осцилляции интенсивности, связанные с рассеянием на слое с отрицательной деформацией, т.е. на слое с параметром решетки, меньшим значения параметра решетки подложки. Эти деформации создают в нарушенном слое дефекты типа вакансий, которые при прочих равных условиях меньше деформируют решетку по сравнению с дефектами междоузельного типа [5].

Используя программный пакет MATLAB, нами были проведены расчеты таких характеристик нарушенного слоя, как $L_{\text {eff }}$ и $\Delta a / a$, непосредственно из экспериментальных данных КДО в соответствии с [4]. 

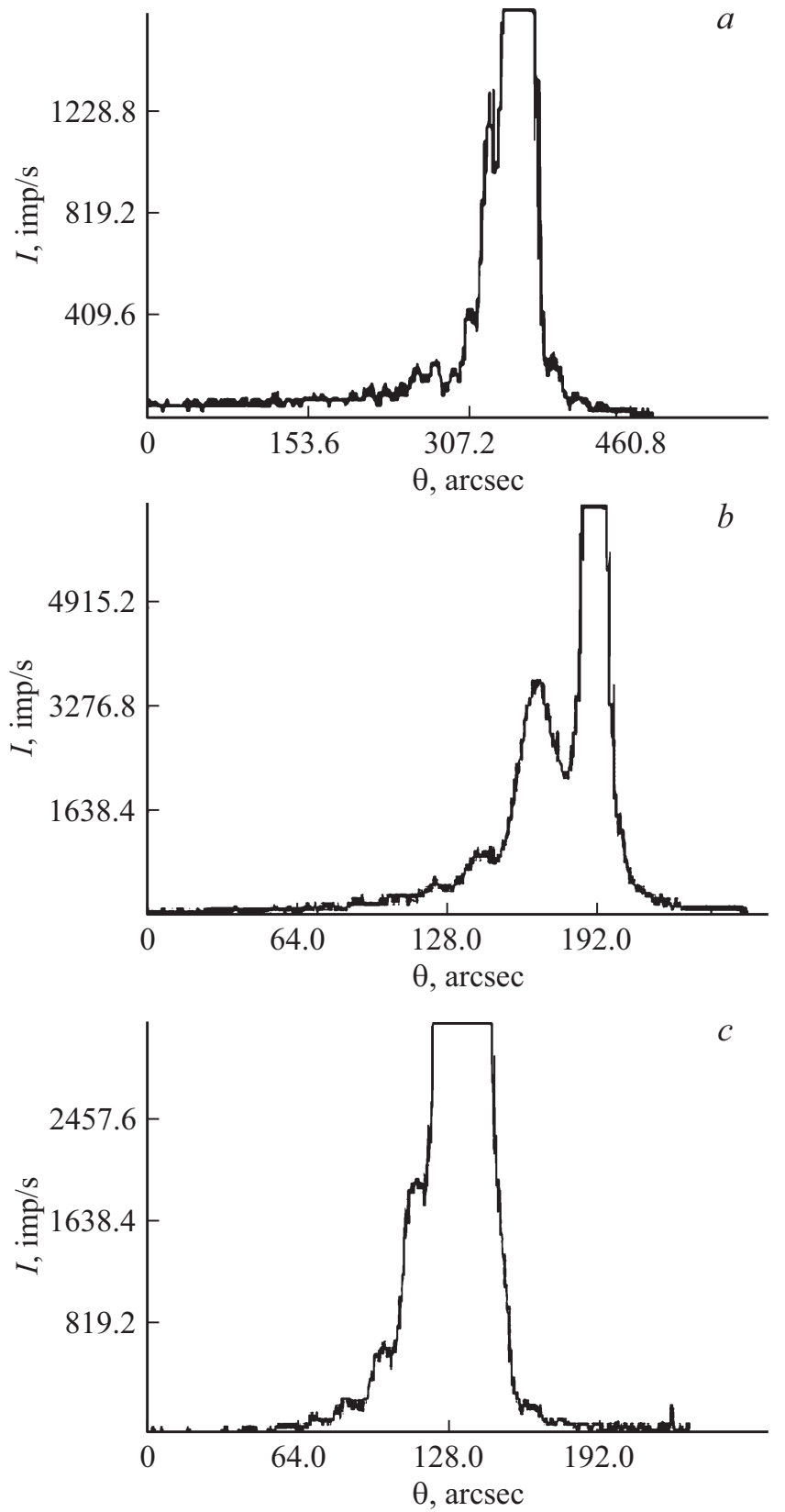

Рис. 3. КДО от образцов $\mathrm{Si}$, облученных протонами с дозой $2 \cdot 10^{16} \mathrm{~cm}^{-2}$ при различных значениях температуры: $a-140$, $b-430, c-610^{\circ} \mathrm{C}$.

При наличии на КДО явно выраженного пика от нарушенного слоя (рис. $2, a, b)$ по угловому расстоянию $\Delta \theta$ между пиками можно определить относительное изменение кристаллических параметров согласно формуле [4]:

$$
\frac{\Delta a}{a}=-\operatorname{ctg} \theta_{\mathrm{B}} \Delta \theta
$$

Если пик от нарушенного слоя в четкой форме не наблюдается, но тем не менее в области малых углов видно заметное увеличение интенсивности отраженных лучей (рис. 2, $c, 3, c)$, которые можно связать с образованием кристаллического слоя с переменным по толщине параметром решетки $a(z)$, то для анализа структуры этого слоя использовался следующий подход. Так как амплитуда рассеяния рентгеновского излучения слоем мала, для интенсивности рассеяния системой слойподложка имеем следующие формулы [4]:

$$
\begin{aligned}
P_{R}(\theta) & =\left|R_{0}(\theta)+R_{1}(\theta)\right|^{2} \\
& =\left|R_{0}(\theta)\right|^{2}+\left|R_{1}(\theta)\right|^{2}+2 \operatorname{Re}\left[R_{1}(\theta) R_{0}^{*}(\theta)\right],
\end{aligned}
$$

где $R_{0}(\theta)$ - амплитуда рассеяния от подложки, $R_{1}(\theta)-$ амплитуда рассеяния нарушенным слоем, которая определяется следующим выражением:

$$
R_{1}(\theta)=-\frac{i}{L_{\mathrm{ext}}} \int_{0}^{L_{d l}} \exp \left\{-w(z)+\frac{i \theta}{\theta_{0}} \frac{L_{d l}-z}{L_{\mathrm{ext}}}-i \mathbf{K}_{h} u(z)\right\} d z .
$$

Здесь $L_{\mathrm{ext}}-$ глубина экстинкции, $L_{d l}-$ толщина нарушенного слоя, $w(z)$ - фактор Дебая-Валлера, характеризующий статическое хаотическое перемещение атомов от их положений в ненарушенном состоянии. Физически фактор $\exp (-w(z))$ учитывает степень аморфизации нарушенного слоя за счет образования в ходе имплантации различного рода дефектов, при этом $\theta_{0}-$ ширина кривой дифракционного отражения, $\mathbf{K}_{h}-$ вектор дифракции, $u(z)$ - смещение атомных плоскостей нарушенного слоя по отношению к их положению в подложке:

$$
u(z)=\int_{0}^{L_{d l}} \Delta a(z) d z
$$

При малых $\Delta a$ размывание пика от нарушенного слоя происходит не только за счет изменения параметров $a(z)$ и $w(z)$ по толщине, но и из-за интерференции амплитуд дифракционного рассеяния от пленки и подложки. Этот процесс описывается в формуле (2) третьим членом. Задавая профили $a(z)$ и $w(z)$, можно, в принципе, рассчитать, в соответствии с формулами (2)-(4), КДО, а соответствующей вариацией этих параметров можно добиться наилучшего согласия расчетных и экспериментальных кривых.

С другой стороны, существует относительно простой метод оценки средних характеристик нарушенного слоя из КДО. Действительно, непосредственно из формул (2) и (3) следует, что разность суммарной площади под КДО от кристалла с нарушенным слоем и от идеального кристалла можно записать в виде $[4,6]$

$$
\Delta S^{(0)}=\frac{1}{\pi} \int_{-\infty}^{\infty} \frac{\left[P_{R}(\theta)-P_{R}^{0}(\theta)\right] d \theta}{\theta_{0}}
$$

и можно представить в виде $[4,6]$

$$
\begin{aligned}
\Delta S^{(0)}=\frac{1}{L_{\mathrm{ext}}} \int_{0}^{\infty} & \{\exp [-2 w(z)-\exp [-w(z)] \\
& \left.\times \cos \left(\mathbf{K}_{h} u(z)\right)\right\} d z
\end{aligned}
$$


Для пленок с относительно большим изменением $\Delta a(z)$ второй член в правой части формулы (5) является малым по сравнению с первым и его можно опустить. Тогда теоретическая формула (5) упрощается

$$
\Delta S^{(0)}=\frac{1}{L_{\mathrm{ext}}} \int_{0}^{L_{d l}} \exp [-2 w(z)] d z .
$$

В случае слабой аморфизации пленки имеем

$$
\Delta S^{(0)} \cong \frac{L_{d l}}{L_{\mathrm{ext}}},
$$

другими словами, величина $\Delta S^{(0)}$ определяет толщину нарушенного слоя.

Далее запишем выражение для первого момента экспериментальной КДО

$$
\Delta S^{(1)}=\frac{2}{\pi \theta_{0}^{2}} \int_{-\infty}^{\infty}\left[P_{R}(\theta)-P_{R}^{0}(\theta)\right] \theta d \theta,
$$

которому соответствует теоретическая формула [6]

$$
\Delta S^{(1)}=\int_{0}^{L_{d l}} \Delta a(z) \exp [-w(z)] d z,
$$

связывающая $\Delta S^{(1)}$ с параметрами нарушенного слоя.

При малой степени аморфизации, когда $\exp (-w(z)) \cong 1$, величина $\Delta S^{(1)}$ определяет среднее отклонение параметра кристаллической решетки нарушенного слоя, а именно

$$
\Delta S^{(1)} \approx \overline{\Delta a(z)} L_{d l} .
$$

Результаты расчетов по формулам (5)-(8) для величин $L_{\mathrm{eff}}(T), \Delta a / a(T), L_{\mathrm{eff}}(D)$ и $\Delta a / a(D)$ для всех измеренных кривых КДО от исследованных образцов приведены на рис. 4, 5 .

Из температурных зависимостей, представленных на рис. 4 , видно, что значения эффективной толщины $L_{\text {eff }}$ и относительной деформации $\Delta a / a$ нарушенного слоя возрастают, достигая максимальных значений при температуре $430^{\circ} \mathrm{C}$. Причем, при ДИ, равной $2 \cdot 10^{16} \mathrm{~cm}^{-2}$, величина $L_{\text {eff }}$ увеличивается в 2.7 раза с ростом температуры $T$, в то время как при меньших дозах она возрастает почти в 4 раза. Относительная деформация $\Delta a / a$ увеличивается в среднем в 1.3 раза при росте температуры $T$ от 50 до $430^{\circ} \mathrm{C}$. Отметим, что при дальнейшем росте температуры $T$ значения величин $L_{\mathrm{eff}}$ и $\Delta a / a$ резко падают.

Из приведенного анализа образования нарушенных слоев в облученных кристаллах кремния можно сделать вывод, что формирование нарушенного слоя определяется не одним, а двумя конкурирующими друг с другом процессами эволюции первичных радиационных дефектов.
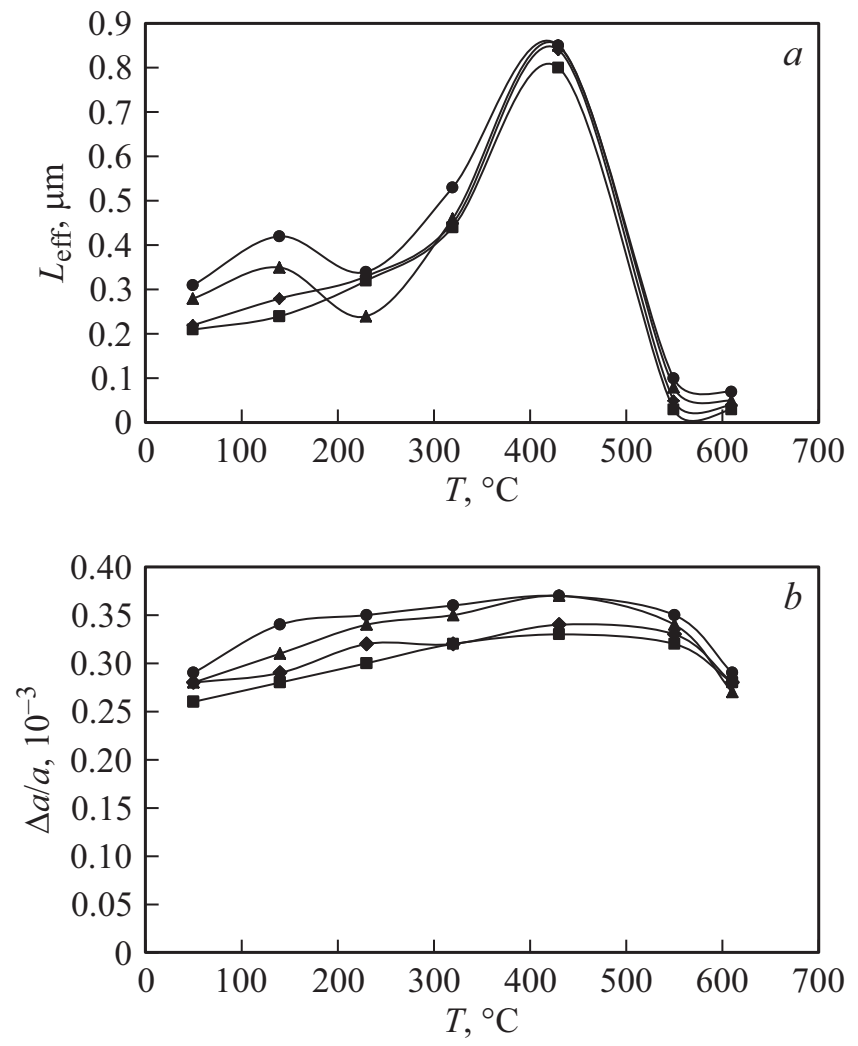

Рис. 4. Температурные зависимости средних значений эффективной толщины нарушенного слоя $L_{\mathrm{eff}}(a)$ и относительной деформации $\Delta a / a(b)$ при разных дозах имплантации (ДИ) образцов кристалла кремния, $D, \mathrm{~cm}^{-2}: \mathbf{\square}-2.5 \cdot 10^{15},-$ $5 \cdot 10^{15}, \mathbf{\Delta}-1 \cdot 10^{16}, \bullet-2 \cdot 10^{16}$.

Так, после выбивания атомов кремния из положения равновесия и образования междоузельных атомов и вакансий имеет место их рекомбинация, что приводит к уменышению концентрации дефектов. С другой стороны, за счет диффузионных процессов первичные междоузельные атомы и вакансии могут удаляться друг от друга и образовывать стабильные комплексы дефектов в виде пар, кластеров и т. п.

Из наших экспериментов следует, что увеличение температуры $T$ до $430^{\circ} \mathrm{C}$ активирует процесс пространственного разделения первичных радиационных пар „междоузельный атом-вакансия“, что приводит к формированию нарушенного слоя на кремниевой подложке.

При дальнейшем увеличении температуры $T$ происходит резкое падение значений величин $L_{\mathrm{eff}}$ и $\Delta a / a$ в результате отжига радиационных дефектов, когда преобладающими становятся рекомбинационные процессы.

Анализ зависимостей $L_{\mathrm{eff}}$ и $\Delta a / a$ от ДИ показывает рост значений этих величин с дозой, причем наибольшие изменения толщины $L_{\mathrm{eff}}$ и относительной деформации $\Delta a / a$ нарушенного слоя от дозы происходят при значениях температуры до $140^{\circ} \mathrm{C}$ в 1.8 и 1.3 раза, соответственно (см. резкий наклон кривых на рис. 5). 

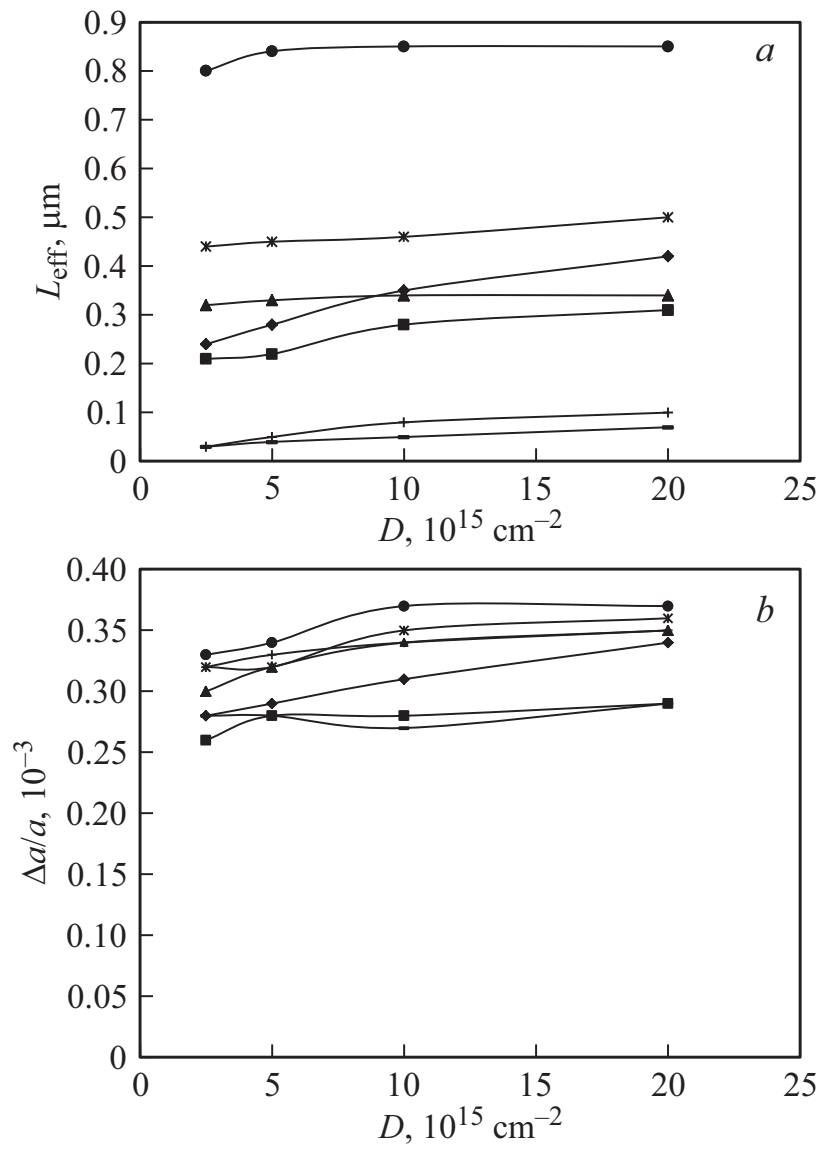

Рис. 5. Дозовые зависимости средних значений эффективной толщины нарушенного слоя $L_{\mathrm{eff}}(a)$ и относительной деформации $\Delta a / a(b)$ при разных температурах $T$ имплантации образцов кристалла кремния, $T,{ }^{\circ} \mathrm{C}: \mathbf{\square}-50, \downarrow-140, \boldsymbol{\Delta}-$ $230, *-320, \bullet-430,+-550,--610$.

Различный наклон кривых дозовой зависимости (рис. 5) позволяет описать образование нарушенного слоя в рамках следующей модели.

Физически ясно, что толщина нарушенного слоя $L_{\mathrm{eff}}$ прямо пропорциональна концентрации исследуемых дефектов. Отсюда следует, что зависимость $L_{\mathrm{eff}}(\mathrm{D})$ можно описать в виде $(i=1,2)$

$$
L_{i}=L_{0 i}\left(1-\exp \left(-\frac{\beta_{i}}{\varphi} D\right)\right)
$$

где $\beta_{i}$ - вероятность отжига радиационных дефектов, $\varphi-$ „скорость“ ввода междоузельных атомов.

В то же время, как это следует из вида кривых на рис. 5, $a$, зависимости $L_{\mathrm{eff}}(D)$ не представляется возможным описать с помощью одной экспоненты. Предполагая, что в зависимости от значения температуры $T$ экспериментально измеренные зависимости $L_{\mathrm{eff}}(D)$ можно представить в виде одной из двух экспоненциальных кривых, отвечающих двум значениям толщины $L_{\mathrm{eff}, 01}$ и $L_{\mathrm{eff}, 02}$, приходим к выводу, что в процессе образования нарушенного слоя могут участвовать не один, а два вида точечных дефектов междоузельного типа. При этом один тип дефектов проявляется при температуре $T=140^{\circ} \mathrm{C}$, в то время как другой при $T=430^{\circ} \mathrm{C}$.

В настоящей работе была предпринята попытка определения энергии активации $E_{a}$ этих дефектов, что дало бы информацию о типе этих дефектов междоузельного типа. Однако набор данных, полученных в наших экспериментах, оказался недостаточным для оценки энергии активации $E_{a}$. Необходимо проведение большего количество экспериментов с больших количество доз облучения.

В случае облучения ионами водорода, помимо „собственных“ радиационных дефектов, в кремнии образуются также радиационные дефекты, в состав которых входят имплантируемые атомы водорода.

Из литературных данных $[7,8]$ известно, что при температурах $T$ до $100-200^{\circ} \mathrm{C}$ в диапазоне доз облучения, меньших $2 \cdot 10^{16} \mathrm{~cm}^{-2}$, практически весь внедренный водород растворен в решетке в составе простых дефектов, к числу которых принадлежат: гидрогенизированные вакансии $\mathrm{VH}_{x=1-4}$ или расщепленные междоузлия $\mathrm{IH}_{x=1,2}$, a также различные комплексы типа $\mathrm{V}_{2} \mathrm{H}_{2}, \mathrm{~V}_{6} \mathrm{H}_{12}$ [9]. Могут также иметь место водородно-примесные центры и молекулы $\mathrm{H}_{2}$, занимающие междоузельные положения в решетке кремния $[10,11]$. Известно, что данные водородсодержащие дефекты обычно распадаются при температурах порядка $200^{\circ} \mathrm{C}$ [12], что косвенно подтверждается нашими экспериментами.

Согласно теории [7], в кремнии, облученном протонами в диапазоне температур $300-450^{\circ} \mathrm{C}$, происходит одновременное высвобождение атомов водорода и их диффузия с захватом на более устойчивых ловушках. В этом отношении захват примеси на ловушках в решетке увеличивает ее эффективную растворимость и приводит к подавлению химического взаимодействия водорода с кремнием, т.е. образования определенных связанных водородных состояний с последующей затем термически стимулированной их перестройки с выделением газа. Подобное взаимодействие приводит к образованию электрически активных дефектов, проявляющих свойства донорных центров. Структура и параметры этих центров зависят от концентрации растворенного водорода.

При температурах выше $450^{\circ} \mathrm{C}$ происходит диссоциация водородных комплексов с одновременным высвобождением атомов водорода. В результате последующей диффузии и десорбции с поверхности водород выделяется из образца. Это приводит к тому, что толщина

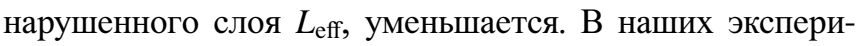
ментах этот факт находит отражение в уменьшении $L_{\mathrm{eff}}$ при температурах выше $430^{\circ} \mathrm{C}$.

При температурах $T$ в диапазоне $500-610^{\circ} \mathrm{C}$ облучение кремния протонами не приводит к образованию в кристалле кремния сильно нарушенного слоя, что объясняется процессами отжига первичных радиационных дефектов. 


\section{Заключение}

В работе приведены и обсуждаются результаты рентгеновского дифракционного исследования различных процессов дефектообразования в кристаллах кремния при облучении их протонами с энергией $150 \mathrm{keV}$ и ДИ $2.5 \cdot 10^{15}-2 \cdot 10^{16} \mathrm{~cm}^{-2}$ в интервале температур от 50 до $610^{\circ} \mathrm{C}$. Из анализа экспериментальных данных с помощью метода КДО определены средние характеристики образующихся нарушенных слоев на подложке кремния: величины эффективной толщины $L_{\mathrm{eff}}$ и относительной деформации $\Delta a / a$ в зависимости от температуры и дозы облучения.

На основании полученных в работе экспериментальных данных было показано, что при изменении ДИ от $2.5 \cdot 10^{15}$ до $2 \cdot 10^{16} \mathrm{~cm}^{-2}$ толщина и деформация нарушенного слоя возрастают примерно в 2 раза. С повышением температуры облучения до $430^{\circ} \mathrm{C}$ эффективные толщина и деформация нарушенного слоя увеличиваются, что обусловлено увеличением числа радиационных дефектов при этих температурах на фоне конкурирующих друг с другом процессов рекомбинации первичных радиационных пар „междоузельный атом-вакансия“ и преобладающим диффузионным разделением их с уходом вакансий в подложку. Показано, что наиболее сильное нарушение поверхности подложки происходит при температуре облучения около $430^{\circ} \mathrm{C}$.

Следует подчеркнуть, что во всех случаях относительные деформации имеют преимущественно положительный знак. Об этом свидетельствует тот факт, что на экспериментальных КДО избыточная отраженная интенсивность, связанная с рассеянием от нарушенного слоя, находится со стороны углов, меньших точного угла Брэгга для подложки. В свою очередь, это согласуется с выводом, что искажение кристаллической решетки поверхностного слоя происходит за счет образования дефектов междоузельного типа. В работе показано, что в формировании нарушенного слоя могут участвовать два типа междоузельных дефектов, образующихся при температурах 140 и $430^{\circ} \mathrm{C}$ соответственно.

Дальнейшее увеличение температуры до $610^{\circ} \mathrm{C}$ не приводит к увеличению толщины нарушенного слоя в кристалле кремния.

Авторы выражают благодарность Н.В. Кузнецову, с.н.с. НИИ ядерной физики МГУ, за облучение образцов на ускорителе КГ-500.

Работа выполнена при поддержке Министерства науки и высшего образования в рамках выполнения работ по Государственному заданию ФНИЦ „Кристаллография и фотоника" РАН.

\section{Список литературы}

[1] Козловский В.В. Модифицирование полупроводников пучками протонов. СПб.: Наука, 2003. 268 с.

[2] Мильвидский М.Г., Освенский В.Б. Структурные дефекты в монокристаллах полупроводников. М.: Металлургия, 1984. 284 c.
[3] Дьячкова И.Г. Формирование нарушенных слоев в кристаллах кремния, имплантированных протонами. Дис. канд. 01.04.07. М., 2004. 172 с. РГБ ОД, 61:04-1/500.

[4] Афанасьев А.М., Александров П.А., Имамов Р.М. Рентгенодифракционная диагностика субмикронных слоев. М.: Наука, 1989. 152 с.

[5] Вавилов В.С., Киселев В.Ф., Мукашев Б.Н. Дефекты в кремнии и на его поверхности. М.: Наука, 1990. 216 с.

[6] Afanas'ev A.M., Kovalchuk M.V., Kov'ev E.K., Kohn V.G. // Phys. Stat. Sol. A. 1977. Vol. 42. P. 415-422.

[7] Александров П.А., Баранова Е.К., Баранова И.В., Бударагин В.В., Литвинов В.Л. Влияние температуры отжига на выход имплантированного водорода из блистеров в кремнии // Труды XII Междунар. совещания „Радиационная физика твердого тела“ (Севастополь, 1-6 июля 2002 г.) / Под ред. Г.Г. Бондаренко. М.: НИИ ПМТ МГИЭМ (ТУ), 2002. C. 149.

[8] Вавилов В.С., Челядинский А.Р. // УФН. 1995. Т. 165. № 3. C. 347-358. [Vavilov V.S. et al. // Phys. Usp. 1995. Vol. 38. N 3. P. 333-343.] DOI: 10.3367/UFNr.0165.199503g.0347

[9] Tokmoldin S.Zh., Mukashev B.N. // Physica B. 2001. Vol. 308-310. P. 167-170.

[10] Leitch A.W.R., Alex V., Weber J. // Phys. Rev. Lett. 1998. Vol. 81. N 2. P. 421-424.

[11] Estreicher S.K. // Mater. Sci. Eng. R. 1995. Vol. 14. N 7-8. P. 319-412.

[12] Watkins G.D. // Mater. Sci. Semicond. Proc. 2000. Vol. 3. P. 227-235. 